\title{
REVISIONES
}

\section{Nuevas tendencias de aprendizaje colaborativo en e-learning. Claves para su implementación efectiva}

\author{
New collaborative learning trends in e-learning. Keys for it effective implementation
Novas tendências de aprendizagem colaborativa em e-learning. Chaves para uma efetiva implementação

\author{
Raidell Avello Martínez ${ }^{1}$, Josep M. Duart ${ }^{2}$ \\ ${ }^{1}$ Universidad de Cienfuegos, Cuba, ravello@ucf.edu.cu, http://ravellom.blogspot.com \\ ${ }^{2}$ Universitat Oberta de Catalunya (UOC), E-Learn Center, España, jduart@uoc.edu
}

\begin{abstract}
RESUMEN
Nos encontramos en el inicio de una nueva evolución, como resultado del desarrollo de las TIC, y su introducción y uso intensivo en las dinámicas sociales y en la realidad educativa. Este avance de la tecnología digital ha creado entornos que favorecen en gran medida la interacción social. En los últimos años han emergido nuevas tendencias en el e-learning como el aprendizaje móvil, el modelo "Flipped Classroom", los MOOC, los entornos personales de aprendizaje, el aprendizaje basado en juegos y las analíticas de aprendizaje; y en todas ellas está presente y se fortalece el aprendizaje colaborativo. Además, se comentaron efectos negativos que puede provocar un uso superficial de actividades colaborativas. Por último, se identificaron aspectos claves para llevar a cabo el diseño de actividades colaborativas, relativos a la formación de los grupos, la selección, claridad, flexibilidad y significatividad de la tarea, y su monitoreo y control.
\end{abstract}

Palabras clave: e-learning, aprendizaje colaborativo, CSCL, TIC, tecnología educativa.

\section{ABSTRACT}

We are at the beginning of a new evolution because of the development of Information Technology and Communication (ICT), and their introduction and intensive use in social dynamics and educational reality. This advance of digital technology has created environments that greatly favor social interaction. In recent years, new trends have emerged in e-learning as mobile learning, the model "Flipped Classroom", massive open online courses, personal learning environments, game-based learning and learning analytics; in all of them, the collaborative learning is strengthened. In addition, some negative effects that can be caused by indiscriminate and superficial use of collaborative activities were discussed. Finally, main issues were identified to be considered to perform the design of collaborative activities, such as those relating to the formation of groups, selection, clarity, flexibility and significance of the task, and monitoring and control.

Keywords: e-learning, collaborative learning, CSCL, ICT, educational technology.

\section{RESUMO}

Encontramo-nos no início de uma nova evolução como resultado do desenvolvimento das Tecnologias da Informação e Comunicação (TICs) e sua introdução e seu uso intensivo nas dinâmicas sociais e na realidade educativa. Este avanço da tecnologia digital tem criado ambientes que, em grande parte, favorecem a interação social. Nos últimos anos, tem emergido novas tendências em e-learning como a aprendizagem móvel, o modelo "Flipped Classroom", os MOOC, os ambientes pessoais de aprendizagem, a aprendizagem baseada em jogos e as análise de aprendizagem; e em todas elas, está presente e se fortalece a aprendizagem colaborativa. Além disso, discutiu-se sobre alguns efeitos negativos que podem causar o uso superficial de atividades colaborativas. 
Finalmente, foram identificadas as principais questões a serem consideradas para o planejamento de atividades colaborativas, relativas à formação de grupos, seleção, clareza, flexibilidade e significância da tarefa e de seu monitoramento e controle.

Palavras-chave: e-learning, aprendizagem colaborativa, CSCL, TIC, tecnologia educacional.

\section{INTRODUCCIÓN}

La forma en que aprendemos y enseñamos avanza en consonancia con la evolución de la civilización humana. Nos encontramos en el inicio de una nueva evolución, como resultado del desarrollo de las Tecnologías de la Información y las Comunicaciones (TIC), y su introducción y uso intensivo en las dinámicas sociales y en la realidad educativa. La educación hoy no puede centrarse en la transmisión del conocimiento, debido a que éste es muy extenso y totalmente accesible gracias a internet. La educación hoy debe centrarse en la preparación de las personas para aprender, para ser autónomas en su proceso de acceso y selección de la información relevante, y para adaptarse a las necesidades cambiantes a lo largo de toda la vida (Cobo y Moravec, 2011). Para ello, el sistema de aprendizaje en "colaboración" es fundamental, y éste es un aspecto todavía en muchos modelos de educación actual.

La disminución del costo de acceso a las tecnologías, así como a la conexión a internet, han superado algunas de las barreras para la adopción de las TIC por parte de todos los actores de la educación, y les han abierto nuevas posibilidades e intereses. De ahí que los docentes y estudiantes deban desarrollar habilidades para responder a las demandas de la sociedad del conocimiento, y sean capaces de procesar y aplicar críticamente la información relevante y pertinente en la resolución de problemas de manera creativa e innovadora. Para responder a este reto, muchas instituciones educativas han adoptado nuevos entornos de aprendizaje que facilitan un aprendizaje flexible y continuo e incorporan la utilización de las herramientas de trabajo colaborativo en red.

El aprendizaje en línea, o e-learning, no es más que una variante del sistema tradicional de educación que pretende resolver la comunicación asíncrona entre estudiantes y profesores. El uso intensivo de internet, el desarrollo de la web social (2.0) y el auge de los dispositivos móviles inteligentes han permitido el desarrollo de nuevas modalidades de educación como la educación en línea, que en ocasiones complementa otras modalidades, como la presencial, y da lugar a la modalidad mixta, o "blended learning". Estas nuevas modalidades híbridas permiten la creación de un continuo formativo que se demuestra muy efectivo en el proceso de aprendizaje (Osorio y Duart, 2011).

Las herramientas de la web social (2.0) han captado la atención de todas las modalidades de educación, debido a que permiten la comunicación entre sujetos, el rápido acceso a la información y la participación colaborativa a diferentes niveles de profesores, estudiantes, así como de toda la comunidad educativa. El uso de todas estas herramientas le ha permitido al e-learning avanzar hacia una dinámica colaborativa que permite la construcción social del conocimiento (Grant, 2011).

El propósito de este artículo es debatir las tendencias que existen en los nuevos escenarios virtuales de enseñanza y aprendizaje con respecto al aprendizaje colaborativo. Y para ello nos cuestionamos: a) ¿En qué medida está presente el aprendizaje colaborativo en las nuevas tendencias del e-learning? y b) ¿Cuáles son los nuevos roles del docente en estos nuevos escenarios? 


\section{NUEVAS RUTAS DEL E-LEARNING Y LA PRESENCIA DEL APRENDIZAJE COLABORATIVO}

El avance del conocimiento como concepto, se ha movido desde el terreno elemental y estático de una perspectiva focalizada en la reflexión individual hasta aquellos determinantes que lo caracterizan como una capacidad dinámica configurada por la participación conjunta de individuos (Suárez y Gros, 2013) .

Las nuevas tendencias, dentro del desarrollo de la tecnología educativa, apuntan hacia un incremento de las actividades basadas en la interacción y la creación colectiva de conocimientos. Estrategias como la resolución de problemas, pensamiento crítico, razonamiento y reflexión pueden ser exitosamente implementadas para la construcción del conocimiento, mediante la creación de entornos de interacción social a través de herramientas para el trabajo colaborativo en red.

En el pasar de los años no se puede decir que el aprendizaje colaborativo se ha redefinido, más bien se ha adaptado a las nuevas herramientas TIC que han surgido, como son la mensajería instantánea, los foros, las wikis, los blogs, los microblogs, las redes sociales, las marcación social, etc. Sobre cada una de estas, o su combinación, se hallaron estudios que se enfocan en demostrar la efectividad del aprendizaje colaborativo a través de ellas.

Dentro de este contexto, la formación virtual, criticada en muchas ocasiones, defendida a ultranza en otras, ha mantenido su desarrollo y se vislumbra un futuro prometedor. Los entornos virtuales de aprendizaje, o aulas virtuales como blackboard, WebCT y Moodle, parecen estar en sus límites, comenzando a complementarse y a extenderse con otras herramientas de trabajo colaborativo en red de gran desarrollo, como las que conforman el entramado web 2.0, como también a adaptarse a los nuevos aparatos como los smartphones y los tablets.

Relacionado con estos últimos, los teléfonos móviles, las tabletas y los ordenadores portables, se encuentra la primera tendencia visible en las investigaciones publicadas en los últimos años, desarrollándose un nuevo concepto: el "aprendizaje móvil" o "mlearning" (Area, 2013). Este enfoque plantea que, cualquier persona conectada a la red puede estar aprendiendo 24 horas al día/7días a la semana desde cualquier lugar o espacio geográfico. El aprendizaje móvil, sigue rompiendo barreras temporales y espaciales que aún no había alcanzado la Educación a distancia. Estar conectado permanentemente permite la interacción constante con los demás estudiantes, profesores y comunidad, como es el caso del uso de twitter, como herramienta para compartir e intercambiar a través de estos dispositivos. El mlearning se ha relacionado también con aprendizaje ubicuo, expandido o invisible.

Gran parte de las investigaciones halladas hacen énfasis en las posibilidades del aprendizaje móvil, por la portabilidad, flexibilidad y el contexto de estas tecnologías, lo cual favorece el aprendizaje, promueve la colaboración y el aprendizaje para toda la vida (Naismith et al, 2004; Dyson et al, 2008; Traxler, 2009; Kim, Lee, y Kim, 2014). En esta línea se encuentra el proyecto The Icollab Project, que propone el uso de dispositivos móviles vinculados al uso educativo de las herramientas de la web 2.0 (Martía, 2011).

Resalta también la investigación de Kim, Lee \& Kim (2014), quienes demostraron, a través de una investigación experimental, las potencialidades de actividades de trabajo colaborativo a través de dispositivos móviles para lograr un aprendizaje significativo. 
Asimismo, se pueden encontrar números monográficos dedicados al aprendizaje móvil en las principales revistas de tecnología educativa, lo cual evidencia la importancia que ha alcanzado esta temática (ET\&S, BJET, etc.). Muy relacionado con esta tendencia se encuentran varias investigaciones que, utilizando la herramienta Twitter, una de las más utilizadas en los dispositivos móviles, han logrado mayor motivación e interacción favorables para el aprendizaje (García y García, 2012; Park, Nam y Cha, 2012; Ryu y Parsons, 2012; Laisema y Wannapiroon, 2014).

Dentro de esta temática, es imprescindible consultar el análisis de las tendencias en el aprendizaje ubicuo y móvil a través de un estudio de las investigaciones publicadas en las principales revistas relacionadas con el e-learning desde el 2001 al 2010 (Hwang y Tsai, 2011), y el meta-análisis llevado a cabo por Wu et al. (2012) con propósito similar.

Otra de las tendencias que se pone en relieve en las investigaciones consultadas, es el "flipped classroom". Este se basa en el trueque de los modelos tradicionales de enseñanza, ofreciendo instrucciones online desde fuera de la clase y trasladando las tareas más prácticas y de interacción social hacia dentro de la clase. El método tradicional representa al profesor como la persona que imparte la clase y orienta deberes para el día siguiente. En este nuevo modelo el profesor permanece a un lado, ejerciendo como guía, mientras que los alumnos trabajan en la clase.

El modelo significa que esta transmisión de los contenidos informativos se realicen empleando las TIC (grabaciones de conferencias en video, lectura de textos, visualización de infografías, presentaciones, mapas conceptuales o murales digitales), de manera que el estudiante pueda acceder a la información en el hogar cuando lo desee en su propio espacio, en constante comunicación con otros alumnos y profesores mediante debates online (Koettgen et al. 2014). De manera similar, define este nuevo enfoque pedagógico, Lowell y Verleger (2013), quienes afirman que emplean grabaciones de las lecturas de clase, en videos, de manera asíncrona así como problemas prácticos a resolver, como tareas para la casa, y por otro lado, las actividades basadas en grupo son realizadas en la clase.

Estos últimos autores, proveen en su estudio más de 150 referencias científicas sobre el enfoque "Flipped Classroom", y a partir de su análisis hallaron características comunes en diferentes dimensiones. Entre otras, fueron estudiadas tipos de actividades tanto en clases como fuera de ella, medidas usadas en las evaluaciones, y características metodológicas de cada estudio. Los resultados muestran que la mayoría de los estudios están basados en exploraciones a grupos únicos; aunque los resultados son balanceados, son generalmente positivos hacia este modelo. Además, encontraron que los estudiantes generalmente prefieren las conferencias presenciales que las grabadas, asimismo prefieren las actividades interactivas ante las conferencias. Igualmente, la mayoría de los estudios sugieren que se mejora el aprendizaje con este modelo, aunque no encontraron mediciones objetivas del aprendizaje. Similares resultados se encuentran en la investigación basada en una consulta (encuesta) a los estudiantes llevada a cabo por Long, Logan y Waugh (2014), quienes indagaron las percepciones de los estudiantes sobre el uso de videos instruccionales en el modelo "Flipped Classroom".

Por otra parte, resaltan los entornos personales de aprendizaje (Personal Learning Enviroment, PLE), referente al conjunto de programas que las personas utilizan para estudiar y aprender de manera autónoma, más allá de lo que puedan aprender dentro del aula con la dirección y supervisión del profesor (Castañeda y Adell, 2013). O sea, las personas con sus entornos personales de aprendizaje no sólo tienen infinidad de recursos 
a su alcance para obtener y procesar información, sino que estos recursos están totalmente fuera del control del profesor (Softic et al. 2013).

Como plantea Area (Area, 2013), el objetivo es que los estudiantes, bien en solitario, bien en colaboración con otros, vayan construyendo de forma procesual y continuada sus propios espacios digitales donde integren aquellos recursos, portales, sitios web, herramientas, o redes que utilizan habitualmente para aprender, comunicarse o desarrollar alguna actividad formativa tanto formal como informal (Cabero, Barroso y Llorente, 2010).

De manera experimental Marín-Juarros, Negre-Bennasar y Pérez-Garcias (2014), pusieron en práctica metodologías didácticas de implementación de PLEs, en una asignatura universitaria de los estudios de maestro de Primaria. Los resultados de la experiencia apuntan a que los alumnos construyen sus PLEs y redes personales de aprendizaje en base a sus nuevos conocimientos adquiridos. Además, como conclusión proponen un modelo de organización metodológica de integración para el aprendizaje colaborativo a modo de buena práctica.

Otra importante tendencia, presente en casi todas las publicaciones y congresos internacionales, son los cursos masivos abiertos en línea: MOOCs (Massive Open Online Courses), derivados de los Recursos Educativos Abiertos (OER) iniciados por el MIT, como el movimiento OCW (McGreal, Kinuthia y Marshall, 2013), y definidos como cursos a distancia pensados para un importante volumen de alumnos, con carácter abierto, global y participativo. Son 'masivos' porque a mayor cantidad de participantes, más posibilidad de encontrar personas afines con las que interactuar, y son 'open' no sólo por la apertura de acceso si no porque no tienen caminos cerrados, uno navega por la materia y los espacios a voluntad, y por ello los resultados de cada uno son distintos (Zapata-Ros, 2013).

En correspondencia con lo anterior, en un reciente estudio de caso publicado por Kellogg, Booth y Oliver (2014), se comentan las deficiencias encontradas en el diseño instruccional y el soporte social en varios trabajos sobre implementación de MOOCs. Ellos proponen y demuestran que con herramientas tan básicas como los foros de discusión, se puede promover el aprendizaje social entre pares. Estos y otros aspectos, como el compromiso con el aprendizaje, diseño y curriculum de los MOOC, aprendizaje autoregulado y social, análisis de redes sociales y la motivación, actitudes y criterios de éxito, son discutidos y analizados en una profunda investigación realizada por Gasevic, Kovanovic, Joksimovic y Siemens (2014).

Así también, en el ámbito iberoamericano se encuentran otros estudios pilotos interesantes (Sosa, López y Díaz, 2014), como el trabajo de Sánchez (2014), donde relata una experiencia en el diseño y producción de cursos MOOC como estrategia de aprendizaje cooperativo en un ambiente de educación a distancia. Para tal efecto, hizo un seguimiento por sesión en los foros de discusión activados en la plataforma Moodle, donde se retrató lo que sucedía en el aula para fortalecer, primero, el aprendizaje colaborativo y, después, el cooperativo.

A partir del análisis de la bibliografía, se puede establecer al "aprendizaje basado en juegos" (Game Based Learning) como una tendencia del e-learning del siglo XXI (Bellotti et al. 2014). A esta tendencia se le otorga un gran potencial para reforzar el aprendizaje en entornos educacionales y de entrenamiento a los juegos digitales. Por ejemplo, Connolly et al. (2012) y Wouters et al. (2013), han publicado dos importantes estudios de la literatura que demuestran esta afirmación. Se pueden encontrar estudios importantes donde se proponen sistemas de evaluación del aprendizaje a partir de los datos generados en los juegos, de los que se infieren habilidades, conocimientos y atributos a los jugadores (Di Cerbo, 2014). 
De igual forma, se encontró un amplio estudio, en correspondencia con el tema de la presente revisión, donde se analizaron 4 programas institucionales llevados a cabo en 18 países europeos, Israel y EEUU, en el período 2002-2009, en el que estuvieron involucrados más de 9.000 estudiantes. Los resultados resaltan una alta efectividad en el desarrollo de competencias transversales, donde sobresalen las colaborativas, con el uso de los juegos tanto de manera remota como presencial (Di Blas y Paolini, 2014).

Por último, no se puede dejar de mencionar una nueva área de investigación que son las "Analíticas de aprendizaje", anunciado en las últimas ediciones del reporte Horizon (2014), donde a partir del gran cúmulo de datos generados de todas las acciones que realiza un usuario en los entornos virtuales de aprendizaje, se pueden extraer valiosos análisis analíticos y gráficos que le pueden brindar al docente una herramienta poderosa para conocer el avance de los estudiantes, así como las tendencias en el aprendizaje, y tomar oportunas decisiones (García-Tinizaray, Ordoñez-Briceño y Torres-Diaz, 2014). Por supuesto que esto incluye todos los datos del trabajo colaborativo que realizan los estudiantes en herramientas como wikis, foros, chats, en video conferencias interactivas, etc.

Así lo definen también Siemens, Dawson, y Lynch (2013), como la colección y análisis de los datos que son generados durante el proceso de aprendizaje, con el propósito de mejorar la calidad de la enseñanza y el aprendizaje. En esta línea se encuentra en trabajo desarrollado en la Purdue University, donde incluso se les provee a los estudiantes de un video donde se les explica que sus datos serán usados para predecir sus resultados y alertarlos sobre problemas potenciales en su progreso (Pistilli et al., 2012).

A pesar de las ventajas comentadas, aludidas a las analíticas de aprendizaje para la mejora del éxito y la retención de los estudiantes sugeridas en muchas investigaciones (e.g., Arnold, 2010; Clow, 2013; Schreurs et al., 2014), Watters (2013) citado por Slade y Prinsloo (2014), alerta que estas suposiciones acerca de los grandes volúmenes de datos "big data" y su relación con el aprendizaje son todavía muy osadas y no probadas.

En correspondencia con estas tendencias bien marcadas en la literatura, se encuentran estudios con resultados más específicos en la aplicación de herramientas para promover el aprendizaje, utilizando estrategias que convergen en el aprendizaje colaborativo, como es el caso de la utilización de Storytelling (Petrucco, 2014), Google Docs (Bonham, 2011), Facebook (Cerdà y Planas, 2011), wikis (DeWitt, Alias y Siraj, 2014), Zotero (Avello et al. 2013), entre otras. Es preciso mencionar que muchos de estos recursos, no sólo se han dirigido e implementado en entornos de aprendizaje formal, más bien tienen un horizonte abierto para cualquier tipo de aprendizaje (Sangrà y Wheeler, 2013).

\section{ALGUNAS REFERENCIAS NEGATIVAS}

A pesar de los beneficios encontrados con el uso del aprendizaje colaborativo en los nuevos escenarios del e-learning, no todo es positivo. Es preciso comentar trabajos donde se describen desventajas o dificultades en la implementación de actividades de colaboración. Para ejemplificar este aspecto, se puede consultar la investigación llevada a cabo por Capdeferro y Romero (2012), donde encontraron que estudiantes de la modalidad educativa en línea pueden encontrar frustrantes las actividades colaborativas y exponen las principales fuentes de frustración encontradas, como son aspectos organizativos del grupo, deficiente distribución de objetivos, participación y calidad de las contribuciones 
desbalanceadas, exceso de tiempo destinado a estas tareas, dificultades en la comunicación, entre otras, que fueron contrastadas con otros estudios (Goold, Craig y Coldwell, 2008; Sallnäs, 2004; Vonderwell, 2003; Jordine, Wilson y Sakpal, 2013).

En este sentido, se encuentra el penúltimo capítulo (9) del reciente libro de Jon Dron y Terry Anderson (2014), donde presentan, como apuntan sus propios autores "la cara oscura del software social". De manera que son analizados temas que deben tener en cuenta quienes pretendan usar el software social para actividades de aprendizaje: como la privacidad, el fraude, efectos imprevistos de la tecnología, desacuerdos culturales, etc.

\section{CLAVES PARA LA IMPLEMENTACIÓN EFECTIVA DEL APRENDIZAJE COLABORATIVO EN EL E-LEARNING}

Más de dos décadas de investigación sobre el aprendizaje colaborativo soportado en computadoras, evidencian efectos positivos en el aprendizaje. Las tecnologías que se han desarrollado, como las herramientas de la web 2.0 y los dispositivos móviles, generan cambios en la dinámica grupal, enriqueciendo las interacciones y creando oportunidades de compartir y construir conocimiento colaborativamente (Järvelä y Hadwin, 2013). Sin embargo, se ha prestado especial atención a aspectos como el número ideal de integrantes del grupo, composición, herramienta usada, sincronicidad, división de la tarea, entre otras, que en ocasiones no son aspectos relevantes para implementar con éxito el aprendizaje colaborativo (Kirschner, Paas y Kirschner, 2009).

A lo largo de la revisión efectuada, se pudieron extraer los aspectos básicos y comunes que han permitido llevar a cabo, con efectividad, actividades colaborativas de aprendizaje dentro de las tendencias del e-learning comentadas con anterioridad. Estos son:

Formación de los grupos. La creación de los grupos parece una tarea fácil en los entornos de e-learning. Sin embargo, se evidenció que para evitar problemas de frustración (Capdeferro y Romero, 2012) y desacuerdos entre los miembros del grupo, es necesario tener en cuenta aspectos claves, como la familiaridad (Janssen et al., 2009) y la empatía (Roberts y McInnerney, 2007). Un diseño efectivo de actividades colaborativas deben tener el propósito bien claro y explícito, aunque se puede brindar un poco de flexibilidad en este sentido, como el poder seleccionar los miembros del grupo y sus roles.

Selección de la tarea grupal. La selección de la tarea es muy importante, porque no todas las tareas son adecuadas para llevarlas a cabo en grupos. Se ha evidenciado que las tareas de mayor complejidad y carga cognitiva son desarrolladas mejor en grupos (Kirschner, Paas y Kirschner, 2009). Sin embargo, en tareas más simples se pierde mucho tiempo en organización y comunicación que no aportan mucho al aprendizaje, y que podría utilizarse en otras tareas de aprendizaje individual.

Claridad y flexibilidad de la tarea. Se debe proveer una guía a los miembros del equipo que explique claramente la tarea, y garantizar que la tarea sea alcanzable en términos de tiempo y esfuerzo. Además, cuando los estudiantes conocen, con detalle, la tarea, como el contenido, proceso, objetivos, consecuencias, resultados esperados, miembros, etc., su compromiso, responsabilidad y pertinencia se incrementa (Juwah, 2006).

Significatividad de la tarea. La significación de la tarea no sólo es vital en entornos virtuales de aprendizaje. Se ha demostrado también en la enseñanza presencial, que la selección de tareas que respondan al contexto y que los estudiantes perciban su utilidad en el futuro 
profesional, le prestan mayor empeño y alcanzan un mayor aprendizaje. Permitirles a los estudiantes que propongan temas acorde a los intereses mútuos de los miembros del grupo, controlar y dirigir su propio aprendizaje, conlleva a una creación colectiva de conocimientos más fluida y efectiva (Lin, 2008). Asimismo, tareas contextualizadas en la realidad del estudiante y utilizando contenidos relevantes puede motivar con mayor fuerza el aprendizaje colaborativo.

Monitoreo y control de la tarea. El docente debe estar disponible para brindar feedback, así como información general y privada durante la mayor parte del proceso colaborativo (Brindley, Walti, y Blaschke, 2009). Además, el docente necesita encausar discusiones que se salgan del tema principal, activar y promover el debate, sobre todo cuando este no se ha suscitado espontáneamente, ayudar en la creación de relaciones y brindar confianza a los estudiantes.

\section{MÁS INFORMACIÓN}

Con el objetivo de indagar con mayor profundidad sobre las tendencias y tecnologías emergentes pueden ser consultadas, permanentemente, las diferentes ediciones de informes muy bien elaborados, como son Horizon Report de New Media Consortium (http://www. nmc.org), A Roadmap for Education Technology del National Science Foundation en los Estados Unidos (http://www.cra.org/ccc/edtech.php ), el reporte de IEEE Technical Committee on Learning Technology (http://www.ieeetclt.org) y el European STELLAR (http://www.stellarnet.eu). Todos estos informes muestras rutas y pistas de las nuevas tendencias o tecnologías emergentes que se están implementando, o próximas a implantarse. Además, brindan las barreras, posibles beneficios, retos de su implantación a corto, mediano y largo plazo.

Resalta en esta línea, un trabajo publicado por Spector (2013), donde integra estos informes, de manera que sintetiza, analiza y compara estas últimas tendencias y tecnologías emergentes, además se sugieren algunas direcciones en la investigación en el área de la tecnología educativa.

De manera más específica, en el campo de la educación a distancia en línea, se encuentra el libro Educación a Distancia en Línea: Hacia una Agenda de Investigación (Online Distance Education: Towards a Research Agenda), que ofrece una panorámica sistemática de los temas más importantes, tendencias y áreas de prioridad en la investigación de la educación a distancia en línea. Para alcanzar este propósito, dos investigadores experimentados en el área de la educación a distancia en general, Olaf Zawacki-Richter y Terry Anderson, invitaron a varios expertos internacionales a abordar cada una de las 15 áreas de investigación identificadas por Zawacki-Richter en un estudio en Delphi llevado a cabo en el 2009 (Zawacki-Richter y Anderson, 2014).

\section{CONCLUSIONES}

El avance de la tecnología digital ha creado entornos que favorecen en gran medida la interacción social. Un gran número de aplicaciones han sido diseñadas e implementadas para soportar el aprendizaje colaborativo, conectando los estudiantes, docentes y comunidad, de manera sincrónica o asincrónicamente, para realizar actividades colaborativas. 
En los últimos años han emergido nuevas tendencias en el e-learning, dentro de las que hemos destacado el aprendizaje móvil, el modelo "Flipped Classroom", los cursos abiertos masivos en línea, los entornos personales de aprendizaje, el aprendizaje basado en juegos y las analíticas de aprendizaje. Después de analizar cada una de ellas, se puede concluir que el aprendizaje colaborativo no ha perdido espacio, más bien se ha fortalecido y adaptado a los nuevos entornos que se han configurado, y está presente como elemento esencial para lograr el aprendizaje en los ciudadanos del siglo 21. Además, se comentaron algunos efectos negativos que puede provocar un uso indiscriminado y superficial de actividades colaborativas.

Por último, teniendo en cuenta lo anterior, se identificaron dentro de la literatura analizada los principales aspectos a tener en cuenta para llevar a cabo el diseño de actividades colaborativas con eficiencia, como los relativos a la formación de los grupos, la selección, claridad, flexibilidad y significatividad de la tarea, y su monitoreo y control.

\section{REFERENCIAS BIBLIOGRÁFICAS}

Area, M. (2012). La formación y el aprendizaje en entornos virtuales. Potencialidades, debilidades y tendencias. Revista Crítica, Noviembre - Diciembre 2012. Consultado 2/10/2014. Recuperado de http://www.revista-critica.com/hacia-donde-va-la-educacion/

Arnold, K. (2010). Signals: Applying academic analytics. EDUCAUSE review. Consultado 2/10/2014. Recuperado de http://www.educause.edu/ero/article/signals-applying-academic-analytics

Avello, R., Martín, I., Díaz, M., y Clavero, M. I. (2013). Zotero, más allá de un gestor bibliográfico. Una experiencia con los docentes y nuevas metas. Revista Didáctica, Innovación y Multimedia, n. 25. Consultado 11/11/2014. Recuperado de http://dim.pangea.org/revistaDIM25/ revista25ARzotero.htm

Bellotti, F., Bottino, R. M., Fernández-Manjón, B., y Nadolski, R. J. (2014). Guest Editorial: Game Based Learning for 21st Century Transferable Skills: Challenges and Opportunities. Educational Technology \& Society, vol.17, n.1, 1-2.

Bonham, S. (2011). Whole class laboratories with Google Docs. The Physics Teacher, vol.49, n.1, $22-23$.

Brindley, J. E., Walti, C. y Blaschke, L. M. (2009). Creating effective collaborative learning groups in an online environment. International Review of Research in Open and Distance Learning, vol.10, n.3, 1-18.

Cabero, J., Barroso, J. y Llorente, M. C. (2010). El diseño de Entornos Personales de Aprendizaje y la formación de profesores en TIC. Digital Education Review, n.18, 27-37.

Castañeda, L. y Adell, J. (2013). Entornos Personales de Aprendizaje: claves para el ecosistema educativo en red. Alcoy: Editorial Marfil

Cerdà, F. L. y Planas, N. C. (2011). Facebook's Potential for Collaborative e-Learning. RUSC. Universities and Knowledge Society Journal, vol.8, n.2, 197-210.

Clow, D. (2013). Looking harder at Course Signals. In Doug Clow's Imaginatively. Blog: New Technology in Higher Education. Consultado 11/11/2014. Recuperado de http://dougclow. org/2013/11/

Cobo, C. y Moravec, J. W. (2011). Aprendizaje invisible. Hacia una nueva ecología de la educación. Barcelona: Col-lecció Transmedia XXI

Connolly, T. M., Boyle, E. A., MacArthur, E., Hainey, T. y Boyle, J. M. (2012). A systematic literature review of the empirical evidence on computer games and serious games. Computers \& Education, vol. 59, n.2, 661-686. 
Estudios Pedagógicos XLII, Nº 1: 271-282, 2016

NUEVAS TENDENCIAS DE APRENDIZAJE COLABORATIVO EN E-LEARNING. CLAVES PARA SU

IMPLEMENTACIÓN EFECTIVA

DeWitt, D., Alias, N. y Siraj, S. (2014). Wikis for Collaborative Learning: A Case Study of Knowledge Management and Satisfaction among Teacher Trainees in Malaysia. Procedia-Social and Behavioral Sciences, n.141, 894-898.

Di Blas, N. y Paolini, P. (2014). Multi-User Virtual Environments Fostering Collaboration in Formal Education. Educational Technology \& Society, vol.17, n.1, 54-69.

Di Cerbo, K. E. (2014). Game-Based Assessment of Persistence. Educational Technology \& Society, vol.17, n.1, 17-28.

Dron, J. y Anderson, T. (2014). Teaching crowds: learning and social media. Athabasca: AU Press

Durall, E., Gros, B., Maina, M., Johnson, L. y Adams, S. (2012). Perspectivas tecnológicas: educación superior en Iberoamérica 2012-2017. Austin, Texas: The New Media Consortium. Recuperado de http://openaccess.uoc.edu/webapps/o2/bitstream/10609/17021/6/horizon_ iberoamerica_2012_ESP.pdf

García Aretio, L. (2014). Bases, mediaciones y futuro de la educación a distancia en la sociedad digital. Madrid: Ed. Síntesis

García, J.L. y García, R. (2012). Aprender entre iguales con herramientas web 2.0 y twitter en la universidad. Análisis de un caso. EDUTEC. Revista Electrónica de Tecnología Educativa, n.40. Consultado 11/11/2014 Recuperado de http://edutec.rediris.es/Revelec2/Revelec40/pdf/ Edutec-e_n40_Garcia_Garcia.pdf

García-Tinizaray, D., Ordoñez-Briceño, K. y Torres-Diaz, J. C. (2014). Learning analytics para predecir la deserción de estudiantes a distancia. Campus virtuales, vol.3, n.1, 120-126.

Grant, M. (2011). Web 2.0 in Teacher Education: Characteristics, Implications and Limitations. En Wired for Learning: An Educator's Guide to Web 2.0, pp. 343-360.

Hwang, G. J. y Tsai, C. C. (2011). Research trend in mobile and ubiquitous learning: A review of publications in selected journal from 2001 to 2010. British Journal of Education Technology, vol.42, n.4, E65-E70.

Janssen, J., Erkens, G., Kirschner, P. y Kanselaar, G. (2009). Influence of group member familiarity on online collaborative learning. Computers in Human Behavior, n.25, 161-170.

Järvelä, S. y Hadwin, A. F. (2013). New frontiers: Regulating learning in CSCL. Educational Psychologist, vol.48, n.1, 25-39.

Juwah, C. (2006). Interactions in online peer learning. En R.C. Sharma y C. Juwah (Eds.) Interactions in online education. Implications for theory and practice. New York: Lawrence Erlbaum, pp. 171-190.

Kirschner, F., Paas, F. y Kirschner, P. (2009). A cognitive load approach to collaborative learning: United brains for complex tasks. Educational Psychology Review, n.21, 31-42.

Koettgen, L., Schröder, S., Borowski, E., Richert, A. y Isenhardt, I. (2014). Flipped classroom on top - excellent teaching through a method-mix. INTED2014 Proceedings, 40-49.

Laisema, S. y Wannapiroon, P. (2014). Design of Collaborative Learning with Creative Problemsolving Process Learning Activities in a Ubiquitous Learning Environment to Develop Creative Thinking Skills. Procedia - Social and Behavioral Sciences, n.116, 3921-3926.

Lin, L. (2008). An online learning model to facilitate learners' rights to education. Journal for Asynchronous Learning Networks, vol.12, n.1.

Long, T., Logan, J. y Waugh, M. (2014). Students' Perceptions of Pre-class Instructional Video in the Flipped Classroom Model: A Survey Study. En M. Searson y M. Ochoa (Eds.), Proceedings of Society for Information Technology \& Teacher Education International Conference 2014. Chesapeake, VA: AACE, pp. 920-927.

Marín-Juarros, V., Negre-Bennasar, F. y Pérez-Garcias, A. (2014). Entornos y redes personales de aprendizaje (PLE-PLN) para el aprendizaje colaborativo. Comunicar, n.42.

Martía, M. C. (2011). Mobile Learning: aproximación conceptual y prácticas colaborativas emergentes. Revista de ciències de l'educació, n.2, 43-50.

McGreal, R., Kinuthia, W. y Marshall, S. (2013). Open Educational Resources: Innovation, Research 
and Practice. Athabasca University. Vancouver: Commonwealth of Learning.

NMC. (2014). Horizon Report 2014. Higher Education Edition. The New Media Consortium. Recuperado de http://www.nmc.org/publications/2014-horizon-report-higher-ed

Osorio, L. A. y Duart, J. M. (2011). A hybrid approach to university subject learning activities. British Journal of Educational Technology, vol.43, n.2, 259-271.

Park, S., Nam, M. y Cha, S. (2012). University students' behavioral intention to use mobile learning: Evaluating the technology acceptance model. British Journal of Educational Technology, vol.43, n.4, 592-605.

Petrucco, C. (2014). Digital Storytelling as a Reflective Practice Tool in a Community of Professionals. Artículo presentado en EDEN Annual Conference 2014.

Pistilli, M.D., Arnold, K. y Bethune, M. (2012). Signals: Using Academic Analytics to Promote Student Success. EDUCAUSE Review Online. Recuperado de http://www.educause.edu/ero/ article/signals-using-academic-analytics-promote-student-success

Roberts, T. S. y McInnerney, J. M. (2007). Seven Problems of Online Group Learning (and Their Solutions). Educational Technology \& Society, vol.10, n.4, 257-268.

Ryu, H. y Parsons, D. (2012). Risky business or sharing the load? Social flow in collaborative mobile learning. Computers \& Education, n.58, 707-720.

Sánchez, M. L. (2014). Diseño y producción de cursos MOOC como estrategia de aprendizaje cooperativo en un ambiente de educación a distancia. Revista Didáctica, Innovación y Multimedia, n.28. Recuperado de http://www.pangea.org/dim/revista28.htm

Sangrà, A. y Wheeler, S. (2013). Nuevas formas de aprendizaje informales: ¿O estamos formalizando lo informal?. En La informalización de la educación [monográfico en línea]. Revista de Universidad y Sociedad del Conocimiento (RUSC). vol.10, n.1, 107-115.

Schreurs, B., de Laat, M., Teplovs, C. y Voogd, S. (2014). Social Learning Analytics applied in a MOOC-environment. eLearning Papers, n.36.

Scott, B. (2011). Whole Class Laboratories with Google Docs. The Physics Teacher, vol.49, n.1, 22-23.

Siemens, G., Dawson, S. y Lynch, G. (2013). Improving the Quality and Productivity of the Higher Education Sector: policy and Strategy for Systems-Level Deployment of Learning Analytics. En Society for Learning Analytics Research, December 2013.

Slade, S. y Prinsloo, P. (2014). Student Perspectives on the Use of Their Data: Between Intrusion, Surveillance and Care. Proceedings of the European Distance and E-Learning Network 2014 Research Workshop. Oxford. 27-28 October, 291-300.

Softic, S., Taraghi, B., Ebner, M., De Vocht, L., Mannens, E. y Van de Walle, R. (2013). Monitoring Learning Activities in PLE Using Semantic Modelling of Learner Behaviour. En Human Factors in Computing and Informatics. Springer Berlin Heidelberg, pp. 74-90.

Sosa, M.J., López, C. y Díaz, S.R. (2014). Comunidad de aprendizaje y participación social en un curso Mooc. International Journal of Educational Research and Innovation (IJERI), n. 1, 1-13.

Spector, J. M. (2013). Emerging Educational Technologies and Research Directions. Educational Technology \& Society, vol.16, n.2, 21-30.

Suárez, C. y Gros, B. (2013). Aprender en red. De la interacción a la colaboración. Barcelona: Editorial UOC

UNESCO. (2012). World Open Educational Resources (OER) Congress 2012 Paris OER Declaration. Recuperado de www.unesco.org/new/fileadmin/MULTIMEDIA/HQ/CI/CI/pdf/Events/English_ Paris_OER_Declaration.pdf

Wiley, D. (2010). OER 101: Theory and Practice. Recuperado de http://opencontent.org/blog/ archives/1725

Wouters, P., van Oostendorp, H., van Nimwegen, C. y van der Spek, E. D. (2013). Meta-analysis of the cognitive and motivational effects of serious games. Computers \& Education, vol.60, n.1, 249-265. 
Wu, W., Wu, Y. J, Chen, C., Kao, H., Lin, C. y Huang, S. (2012). Review of trends from mobile learning Studies: A meta-analysis. Computers \& Education, vol.59, n.2, 817-827.

Zapata-Ros, M. (2013). MOOCs, una visión crítica y una alternativa complementaria: La individualización del aprendizaje y de la ayuda pedagógica. Revista Campus Virtuales, vol.2, n. $1,20-38$.

Zawacki-Richter, O. (2009). Research Areas in Distance education: A Delphi study. International Review of Research in Open and Distance Learning, vol.10, n.3. Recuperado de http://www. irrodl.org/index.php/irrodl/article/view/674/126

Zawacki-Richter, O. y Anderson, T. (2014). Online Distance Education: Towards a Research Agenda. Athabasca: AU Press 